\title{
A relação entre renda e composição domiciliar dos idosos no Brasil: um estudo sobre o impacto do recebimento do Benefício de Prestação Continuada
}

\author{
Maira Andrade Paulo* \\ Simone Wajnman ${ }^{* *}$ \\ Ana Maria Camilo Hermeto de Oliveira ${ }^{\star *+}$
}

Este trabalho analisa o impacto do recebimento do Benefício de Prestação Continuada (BPC) sobre a composição dos arranjos domiciliares dos idosos pobres no Brasil. O BPC corresponde ao benefício mensal no valor de um salário mínimo (SM) destinado a idosos acima de 65 anos, cuja renda familiar per capita seja inferior a 1/4 de SM. Essa discussão é bastante oportuna no atual contexto brasileiro, que vem sendo marcado por um acelerado envelhecimento populacional, por profundas mudanças nos arranjos domiciliares e pela ampla cobertura de seguridade social do idoso. Utilizando o método estatístico da "diferença em diferença" e os dados da PNAD de 2002 e 2004, são investigadas duas hipóteses discutidas na literatura sobre o tema: o recebimento de uma transferência incondicional de renda elevaria a probabilidade de os idosos viverem sozinhos, em função da autonomia conferida pela renda, ou aumentaria a probabilidade da corresidência com familiares devido à atração exercida pela renda. 0 resultado sugere a hipótese de que o recebimento do BPC, no Brasil, tem aumentado a probabilidade de formação de domicílios unipessoais.

Palavras-chave: Arranjos domiciliares dos idosos. Transferência de renda. Benefício de Prestação Continuada (BPC).

\footnotetext{
* Universidade Salgado de Oliveira, Belo Horizonte-MG, Brasil (mairapaulo@gmail.com).

${ }^{\star \star}$ Departamento de Demografia da Universidade Federal de Minas Gerais - Cedeplar/UFMG, Belo Horizonte-MG, Brasil (wajnman@cedeplar.ufmg.br).

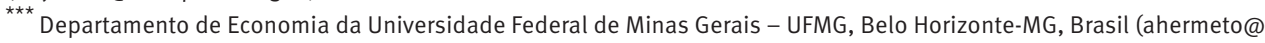
cedeplar.ufmg.br).
} 


\section{Introdução}

A composição dos domicílios é essencial na determinação do bem-estar das pessoas. No que diz respeito aos idosos, os arranjos domiciliares podem influenciar o funcionamento das redes de suporte privado, assim como a dinâmica das contribuições financeiras dos idosos direcionadas a familiares mais desprovidos de renda. No atual contexto de envelhecimento populacional, mudanças nas famílias e políticas públicas de apoio aos idosos, é importante a compreensão da dinâmica de formação dos arranjos domiciliares dos idosos. Dessa forma, o artigo busca investigar como o recebimento de uma renda extra, no caso o Benefício de Prestação Continuada (BPC), pode ter impactado a composição dos arranjos desse segmento populacional no Brasil.

Entre os determinantes dos arranjos domiciliares dos idosos, a renda tem papel de grande destaque (CAMARGOS et al., 2007; SAAD, 2000; GHOSH, 2007; MARTELETO, 2007), ${ }^{1}$ sendo que duas possibilidades são apontadas na literatura: o recebimento de uma renda pode, por um lado, estimular os idosos a buscarem independência, optando por morarem sozinhos (COSTA, 1999; MCGARRY; SCHOENI, 1998; CARVALHO, 2000) e, por outro, principalmente em situações de pobreza, atrair familiares interessados em compartilhar desses benefícios, aumentando a probabilidade da coabitação (EDMONDS et al., 2005; SAAD, 2000; CAMARANO, 2003; CIOFFI, 1998). O propósito deste trabalho é testar qual desses efeitos predomina quando ocorre um choque exógeno de renda na forma do recebimento, pelo idoso, de um benefício social antes inexistente.

Os idosos brasileiros - com 60 anos e mais -, que até 1980 representavam aproximadamente $6 \%$ do total da população, em 2020 responderão por cerca de $13 \%$ e, em 2050, chegarão a 29\% da população (IBGE, 2008). Em termos de seguridade social, os idosos brasileiros vêm conquistando cada vez mais direitos. A Constituição brasileira de 1988 buscou garantir direitos mínimos a uma população que envelhece rapidamente e que, em grandes proporções, encontra-se em situações adversas de pobreza. O acesso dos idosos a benefícios sociais foi universalizado, seja na forma de benefícios previdenciários, seja na forma de transferências de renda sem vínculo contributivo. No meio rural, os idosos que comprovam qualquer tipo de trabalho agrícola de subsistência passaram a ter acesso a beneficio de uma aposentadoria no valor de um salário mínimo. Na área urbana, instituiuse, a partir de 1996, o Beneficio de Prestação Continuada (BPC), que é uma transferência não contributiva de renda, no valor de um SM, destinado a idosos não protegidos pelas aposentadorias e que comprovem possuir renda familiar per capita $(\mathrm{rfpc})$ inferior a $1 / 4 \mathrm{de} \mathrm{SM}$.

Tendo em vista tais fatores, este trabalho procura investigar especificamente o impacto do Benefício de Prestação Continuada sobre a composição dos arranjos domiciliares dos idosos no Brasil. De acordo com o Anuário Estatístico do Ministério da Previdência Social, desde a implementação do BPC, o número de benefícios concedidos aumentou de aproximadamente

\footnotetext{
1 Além da renda, a condição de saúde dos idosos (GRUNDY, 2000; RAHMAN, 2000) e o tamanho e a composição da rede de parentes, em especial filhos (MARTELETO, 2007; MCGARY; SCHOENI, 1998), são outros determinantes importantes dos arranjos domiciliares dos idosos destacados pela literatura.
} 
50.000, em 1996, para quase 1 milhão e meio, em 2006 (BRASIL, 2006), de tal forma que $10 \%$ da população brasileira acima de 60 anos passou a receber este benefício. A proposta deste trabalho é verificar se o recebimento dessa transferência de renda, antes inexistente, aumentou a propensão dos idosos a morarem sozinhos ou se, ao contrário, provocou o aumento da coabitação de familiares em torno dos beneficiários.

Estudos realizados acerca da relação entre renda e arranjo domiciliar dos idosos apresentam resultados que merecem atenção. Nos EUA, Costa (1999) mostrou que a institucionalização do recebimento de pensão (Old Age Assistance), na década de 1940, foi coincidente com o início da tendência de crescimento na proporção de mulheres idosas morando sozinhas. Até esse período, a maioria das idosas vivia com filhos e outros parentes. A partir de 1940, verificou-se uma expressiva diminuição dessa situação, sendo que, no final da década de 1990, apenas uma minoria das idosas vivia com filhos e outros parentes. Na mesma direção, Engelhardt et al. (2005), utilizando mudanças legislativas nos benefícios previdenciários de seguridade social dos EUA, constataram que reduções no benefício tiveram impacto significativo, no sentindo de fazer migrar os idosos que viviam em arranjos domiciliares independentes para arranjos com mais pessoas.

Por outro lado, Edmonds et al. (2005), ao estudarem como a composição domiciliar responde à mudança na renda domiciliar na África do Sul, não encontram evidências de que um crescimento da renda provoca aumento da propensão de o idoso morar sozinho; ao contrário, o estudo enfatiza o papel do idoso em dar suporte a suas famílias. Uma explicação plausível para os resultados obtidos, segundo os autores, é a relação entre pobreza e famílias estendidas. Na mesma linha, estudo comparativo entre viúvos de diferentes regiões brasileiras sobre o efeito da renda nos arranjos domiciliares dos idosos apontou que a alta renda do idoso, no Sudeste, separa os domicílios, enquanto a pobreza estimula a corresidência, no Nordeste, indicando que a preferência do idoso pode ser sobrepujada pelas necessidades dos filhos adultos (SAAD, 2000).

Previamente à investigação do impacto do recebimento do BPC sobre o arranjo domiciliar dos idosos, no presente artigo analisa-se a evolução dos arranjos domiciliares deste subgrupo em 1985, 1995 e 2005, segundo quartis de renda, utilizando dados das PNADs (Pesquisa Nacional por Amostra de Domicílios). Essa análise revela a importância do recebimento de uma renda no valor de um SM na configuração do arranjo domiciliar do idoso. Em 2005, 2,5\% dos idosos e idosas com renda inferior a um SM per capita viviam sozinhos, proporção que salta para $27,3 \%$ no quartil de rendimento seguinte, em que se encontra o recebimento no valor de um SM per capita. Apesar de, nesse caso, o recebimento de um SM poder estar associado à aposentadoria, à aposentaria rural, ao BPC, ou a qualquer outra renda no valor de um SM, esse resultado fornece suporte para a investigação do impacto do BPC sobre o arranjo domiciliar do idoso, principalmente quando se consideram os desafios metodológicos associados às avaliações de impacto de políticas sociais. Para a investigação do impacto do BPC sobre o arranjo domiciliar, tirou-se proveito da mudança na legislação referente ao recebimento do BPC, ocorrida em 2003, que reduziu a idade mínima de elegibilidade de 67 
para 65 anos. Essa alteração pode ser vista como um choque exógeno de renda aos idosos que passaram a se beneficiar antes do previsto, podendo ser entendida, assim, como uma descontinuidade do recebimento do BPC, que fornece um experimento quase-natural para avaliação do impacto que o artigo se propõe a estudar (MIRANDA, 2008).

Para a verificação empírica das hipóteses, foram utilizados o método estatístico da diferença em diferença e os dados da PNAD de 2002 e 2004. A estratégia adotada para identificar o impacto do BPC sobre o arranjo domiciliar dos idosos é comparar o grupo de idosos que foram afetados pela mudança (não recebiam beneficio e passaram a ter o direito de recebimento devido à alteração na legislação) com o grupo não afetado por essa mudança. O resultado obtido com o exercício proposto sugere que o recebimento do benefício aumentou, entre 2002 e 2004, a probabilidade de o idoso chefe ou cônjuge morar sozinho.

Na seção que se segue, é apresentada a metodologia da análise de impacto do BPC sobre as variáveis indicadoras de composição domiciliar. Posteriormente, analisa-se a evolução dos arranjos domiciliares dos idosos no Brasil nas duas últimas décadas, segundo um recorte de renda. São expostos os resultados do exercício metodológico do impacto da renda sobre a determinação do arranjo domiciliar dos idosos e, por fim, apresentam-se as considerações finais do trabalho.

\section{Fonte de dados e método de análise}

\section{Fonte de dados}

Neste estudo, foram utilizadas as bases de dados da Pesquisa Nacional por Amostra de Domicílios (PNAD) do IBGE, que fornecem informações acerca dos arranjos domiciliares e familiares, com uma grande diversidade de variáveis socioeconômicas, além de dados sobre a renda proveniente do BPC, o que permite investigar o assunto proposto.

Vale notar que a PNAD de 2004 apresenta um suplemento sobre programas de transferências de renda e investiga especificamente os beneficiários do programa BPC. No entanto, Soares et al. (2006), ao compararem as informações da PNAD sobre recebimento dos programas de transferência de renda com os dados administrativos do governo federal, verificaram que a cobertura da primeira é bem inferior aos dados administrativos. Enquanto, em 2004, segundo o Ministério do Desenvolvimento Social, o registro do BPC destinado ao idoso correspondia a 885.236 benefícios, na PNAD o número (expandido) de beneficiários assim declarados foi de 324.575, ou seja, $60 \%$ inferior aos dados oficiais. Soares et al. (2006) apresentaram dois motivos para essa diferença: a montagem amostral da PNAD, que é definida com o Censo Demográfico que abre a década, ou seja, o peso e a amostra são definidos antes da PNAD ir a campo; e os erros de declaração por parte da população idosa ou daqueles que respondem o questionário, ao confundir o BPC com aposentadoria ou pensão. A PNAD de 2002 não apresenta suplemento de transferência de renda. 
Tendo a qualidade duvidosa dessa informação contida no suplemento da PNAD de 2004, optou-se por identificar o recebimento do BPC de forma alternativa, conforme se detalha a seguir.

\section{Método de análise causal do impacto do recebimento do BPC sobre o arranjo domiciliar}

A estratégia metodológica para análise causal do impacto do recebimento do BPC sobre o arranjo domiciliar aproveitou-se do fato de que, após sua implementação, o BPC passou por mudança na legislação, que reduziu a idade mínima para a elegibilidade - que correspondia a 67 anos desde 1998 - para 65 anos, a partir de 1ํ de janeiro de 2004, por determinação do Estatuto do Idoso da Lei n. 10.741 (BRASIL, 2003).

Usando a estratégia sugerida e utilizada por Carvalho Filho (2000), bem como por Miranda (2008), empregou-se a mudança da legislação, ocorrida em 2004, como forma de avaliar o impacto do BPC sobre o arranjo domiciliar dos idosos. O trabalho de Carvalho (2000) usou a alteração na legislação acerca da idade limite ao recebimento da aposentadoria rural para avaliar seu impacto sobre os arranjos domiciliares, enquanto Miranda (2006) valeu-se da mudança na idade limite para o recebimento do BPC para analisar o efeito de transferências privadas interdomiciliares.

Nessa estratégia, os idosos de 63-64 anos são tomados como grupo de controle e aqueles com 65-66 anos como grupo de tratamento. Isto porque, antes de 2004, os idosos em ambas as faixas etárias não recebiam o BPC, uma vez que a idade mínima para a elegibilidade era de 67 anos; a partir de 2004, os idosos de 65-66 anos tornam-se elegíveis para o recebimento do benefício, enquanto o grupo de 63-64 anos permanece sem esse direito. Diante dessa mudança, comparam-se as diferenças nas variáveis indicadoras da composição domiciliar (explicitadas adiante) entre os idosos desses dois grupos de idade, em 2002 e 2004. A diferença entre essas diferenças dará o impacto da mudança na legislação sobre os arranjos dos idosos. Considera-se que qualquer diferença existente entre o grupo de 63-64 anos e o de 65-66 anos, em 2002, pode ser explicada pela idade. Em 2004, a diferença entre os grupos devido à idade se mantém, mas a ela é acrescido o impacto do recebimento do BPC. Portanto, da diferença observada entre esses grupos antes e depois de 2004, obtém-se o efeito do recebimento do BPC sobre os arranjos domiciliares. ${ }^{2}$ A Figura 1 ilustra a comparação entre os grupos controle e de tratamento. Em 2002, comparam-se os idosos de 63-64 anos e 65-66 anos, na situação em que ambos os grupos não tinham direito ao benefício; já em 2004, a comparação ocorre na situação em que os primeiros não tinham direito ao benefício, enquanto os últimos passam a tê-lo a partir desse ano.

\footnotetext{
${ }^{2}$ Não se pode deixar de destacar que o período analisado é curto e, portanto, possíveis mudanças nos arranjos podem ter ocorrido em período posterior, não sendo captadas nesse exercício.
} 
FIGURA 1

Visualização da comparação na análise do método “diferença em diferença”

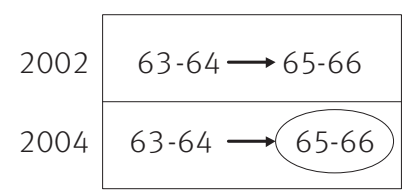

O método utilizado é o da diferença em diferença (DD), que é bastante empregado na avaliação de políticas públicas quando se possui informação relativa a antes e depois do período de implementação de políticas. Considerando um grupo de controle e outro de tratamento, a ideia do método consiste em verificar a mudança na variável de interesse entre os dois grupos antes e depois do impacto da política, sendo que, da diferença dessas diferenças (dupla diferença) verificada, obtém-se o resultado estimado do impacto do programa sob a variável de análise, ou, em outras palavras, o efeito médio do tratamento no tratado.

Utilizando A e D para denotar “antes" e "depois" da intervenção, a mudança média na variável de interesse $Y$, no grupo de tratamento, é estimada por $E\left(Y_{D}-Y_{A} / T=1\right)$ e, no de controle, por $E\left(Y_{D}-Y_{A} / T=0\right)$.

onde:

- Y é a variável resposta;

- $T$ é a variável tratamento ou controle, sendo $T=0$ (grupo controle) e $T=1$ (grupo tratamento).

O resultado estimado da diferença em diferença (DD) é dado por:

$D D=E\left(Y_{D}-Y_{A} \mid T=1\right)-E\left(Y_{D}-Y_{A} \mid T=0\right)$

As estimativas do efeito médio do tratamento, utilizando o método da diferença em diferença, podem ser calculadas tanto por meio do uso de médias simples - como mostrado na equação (1) - quanto via regressão - conforme a equação (2) que se segue.

É estimado um modelo logito, pois as variáveis respostas são categóricas dicotômicas:

Logit $\mathrm{Yi}=\beta_{0}+\beta_{1} \mathrm{Pi}+\beta_{2} \mathrm{Ti}+\beta_{3}\left(\mathrm{Pi}^{\star} \mathrm{Ti}\right)+\beta_{4} \mathrm{Xi}+\varepsilon \mathrm{E}$ onde:

- Yi: variável resposta (indicadora da composição domiciliar);

- Pi: variável de período (assume valor 0 se o ano corresponder a 2002 e valor 1 se o ano for 2004);

- Ti: variável de tratamento (assume valor 0 se grupo de controle corresponder a 63-64 anos e valor 1 se grupo de tratamento corresponder a 65-66 anos);

- $\mathrm{Pi}^{\star} \mathrm{Ti}$ : variável de interação;

- Xi: variáveis de controle.

- Ei: termo do erro

O coeficiente da variável de interação $\left(\beta_{3}\right)$ fornece o resultado da análise do impacto do recebimento do BPC sobre as variáveis respostas analisadas - indicadoras de composição 
domiciliar. O resultado da magnitude do coeficiente da interação é apresentado em termos da razão das razões de chance (odd odds ratio), uma vez que se trata de dupla diferença.

$$
\beta_{3}=\ln \left(\frac{\frac{\text { odds para Trat }=1, \text { Periodo }=1}{\text { odds para Trat }=0, \text { Período }=1}}{\text { odds para Trat }=1, \text { Período }=0}\right)
$$

A interpretação dessa razão das razões de chance corresponde ao diferencial líquido, ou seja, líquido do efeito de período e das diferenças estruturais entre os grupos.

Em termos da razão das razões de chance, os coeficientes encontrados devem ser interpretados como a chance de os indivíduos do grupo de controle terem uma probabilidade de experimentar o evento medido pela variável resposta maior ou menor do que os indivíduos do grupo de tratamento.

Deve-se deixar claro que o resultado do termo da interação corresponde à diferença entre os grupos analisados (63-64 anos e 65-66 anos) entre os períodos considerados (2002 e 2004), ou seja, capta-se o adicional da diferença entre os grupos (63-64 anos e 65-66 anos) ao longo dos anos (2002 e 2004). Em outras palavras, busca-se o resultado líquido do impacto ao eliminar as diferenças existentes entre os grupos e ao longo do período.

As variáveis respostas indicadoras da composição domiciliar selecionadas foram:

- idosos(as) chefes que moram com familiares (sejam eles cônjuges, filhos ou outros parentes de qualquer idade). Essa variável corresponde ao oposto de morar sozinho e assume valor 0 se o idoso mora sozinho; e 1 se o idoso mora com familiares;

- idosos(as) morando na presença de filhos menores de 21 anos em domicílios cujo chefe ou cônjuge são idosos - a variável assume valor 0 se não há filhos; e 1 se há filhos no domicílio;

- idosos(as) morando na presença de outros parentes de qualquer idade em domicílios cujo chefe ou cônjuge são idosos - a variável assume valor 0 se não há outros parentes no domicílio; e 1 se há algum outro parente no domicílio;

- tamanho do domicílio menor ou maior que três habitantes em domicílios cujo chefe ou cônjuge são idosos - a variável assume valor 0 se o domicílio tem 1 a 3 habitantes; e 1 se o domicilio tem mais de três habitantes.

O método da DD ainda permite incluir variáveis de controle na regressão (Xi), servindo para garantir que a comparação entre os grupos de idosos de 63-64 anos com os de 6566 anos, por exemplo, se dê entre indivíduos com características parecidas, tais como escolaridade, sexo, localização do domicílio, etc. As variáveis de controle utilizadas foram:

- variáveis individuais

- sexo (0 para mulher; 1 para homem);

- raça (0 para raça/cor branca ou amarela; 1 para preta, parda ou indígena);

- escolaridade (anos de estudos);

- condição da união (0 para ausência de cônjuge no domicílio; 1 para presença de cônjuge no domicílio);

- variáveis de infraestrutura da unidade domiciliar 
- paredes (0 se material predominante das paredes externas é de taipa não revestida, madeira aproveitada, palha ou outro material; 1 se parede externa é de alvenaria ou madeira aparelhada);

- telhado (0 se material predominante na cobertura - telhado - é de zinco ou madeira aproveitada ou palha; 1 se telhado é telha ou laje de concreto ou madeira aparelhada);

- banheiro (0 se tem banheiro ou sanitário no domicílio; 1 se não tem banheiro no domicilio);

- água encanada (0 se não tem água canalizada em pelo menos um cômodo; 1 se tem água canalizada);

- lixo (0 se o lixo domiciliar é jogado em terreno baldio, logradouro, rio ou mar; 1 se coletado diariamente, indiretamente, queimado ou enterrado na propriedade);

- iluminação (0 se forma de iluminação do domicilio é óleo, querosene, gás de botijão ou outra forma; 1 se elétrica - de rede, gerador ou solar);

- variáveis de localização do domicílio

- situação censitária (0 se situação censitária do domicílio é rural; 1 se urbano);

- região metropolitana (0 se região não é metropolitana; 1 se região é metropolitana);

- grande região (0 se domicílio está na Região Norte, Nordeste ou Centro-Oeste; 1 se domicilio está na Região Sul ou Sudeste).

Ainda é importante destacar que somente os idosos com renda per capita abaixo de 1/4 de SM entraram na análise, uma vez que é este o critério para recebimento do BPC. Com o recebimento desse benefício, os idosos provavelmente passam a ter uma renda familiar per capita superior a $1 / 4$ de SM, sendo necessário, por esse motivo, descontar o valor referente a um SM da renda familiar daqueles que se atribuíram o recebimento do BPC, de forma a possibilitar sua identificação. Trabalhou-se, portanto, com a renda descontada do recebimento do BPC, ou seja, a renda líquida do valor do BPC. Vale dizer que o BPC considerado corresponde ao recebimento de exatamente um SM na variável "outros rendimentos" da PNAD (SOARES et al., 2006). Além do BPC, nessa variável encontram-se também declarados os juros de caderneta de poupança e de outras aplicações, dividendos e/ou outros rendimentos que o indivíduo recebia no mês de referência, assim como os rendimentos referentes aos programas de transferência de renda. No entanto, o BPC é uma renda que se destaca pelo valor exato de um SM.

Uma vez identificados os idosos com renda familiar per capita inferior a $1 / 4$ de SM, selecionaram-se aqueles que, de acordo com os critérios de elegibilidade, não recebem benefício previdenciário (os que declararam não ter recebimento de aposentadoria e pensão). Os idosos que declararam receber entre 0 e menos de um SM de aposentadoria ou pensão também foram incluídos no grupo analisado, pois considerou-se que valores inferiores a um SM de aposentadoria ou pensão não impediriam que o idoso requeresse o BPC.

Por haver erros de declaração quanto ao recebimento do BPC, uma vez que muitos idosos erroneamente declaram esse benefício como aposentadoria (SOARES et al., 2006), optouse, como sugerido por Miranda (2008), por incluir, no conjunto de possíveis recebedores do BPC, os idosos que declararam receber o valor exato de um SM de aposentadoria. Ao 
tomar essa decisão, o grupo de recebedores do BPC é inflado com aqueles que recebem aposentadoria, o que poderia alterar a análise. Para lidar com esse problema, não somente os idosos de 65-66 anos recebedores de um SM no ano posterior à mudança na legislação foram considerados, mas sim todos aqueles, nas duas faixas etárias, que recebiam, antes e depois da mudança, um SM de aposentadoria. Dessa forma, o recebimento de um SM de aposentadoria exerce influência no arranjo domiciliar desses dois grupos. No entanto, para os idosos de 65-66 anos depois da mudança, ou seja, aqueles que de fato recebiam o BPC, o efeito medido corresponde à soma tanto do BPC quanto da aposentadoria erroneamente declarada, o que leva, quando se comparam os grupos por meio da diferença em diferença, a captar o efeito exclusivo do recebimento do BPC.

Para o cálculo da renda familiar per capita de elegibilidade para o BPC, foram contabilizados os rendimentos do idoso, do seu cônjuge e dos filhos menores de 21 anos, sendo todos residentes no domicílio. A renda individual engloba todos os rendimentos previdenciários, os de trabalho, as transferências governamentais e interdomiciliares do indivíduo.

Uma vez bem definidos os grupos de controle e tratamento, parte-se para a análise da composição domiciliar desses indivíduos. Entre os idosos, as posições no domicílio consideradas para análise foram as de chefe, cônjuge, filho ou outro parente. Destas, excluíram-se a posição de filho no domicílio, em função dos poucos casos verificados, e a de outro parente, pois, apesar de o número de casos ser expressivo, esbarra-se na dificuldade em identificar a exata relação de parentesco entre eles e os outros membros familiares (visto que poderiam ser pais, sogro, tio, irmão, etc.). Assim, a análise econométrica concentra-se apenas em verificar o impacto do recebimento do BPC sobre os idosos chefes ou cônjuges do domicílio. Trata-se de uma limitação, mas entre as possibilidades, considerou-se a melhor solução.

A seguir, é apresentada uma análise puramente descritiva dos arranjos domiciliares dos idosos ao longo dos anos de 1985, 1995 e 2005, de fundamental importância para a compreensão dos resultados referentes à análise do impacto do BPC sobre os arranjos domiciliares dos idosos.

\section{Composição dos arranjos domiciliares dos idosos e sua relação com a renda, em 1985, 1995 e 2005}

Esta seção traz a relação da composição dos arranjos dos idosos com renda para 1985, 1995 e 2005. Os dados utilizados são da PNAD dos respectivos anos. Foram selecionados todos os idosos que assumem a posição de chefe, cônjuge ou outro parente no domicílio, distribuídos por quartil de renda.

A Tabela 1 elucida bastante a questão dos arranjos domiciliares dos idosos e sua relação com a renda. Chama a atenção o forte aumento na proporção de domicílios do tipo idoso

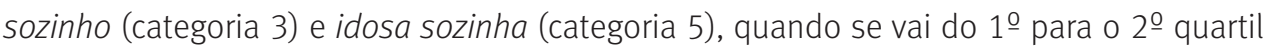


de renda, em qualquer um dos três anos analisados. Tomando-se 2005 como exemplo, verifica-se que a proporção de idoso sozinho salta de 1,3\% para 9,2\%, entre esses dois quartis de renda, variação que é ainda mais expressiva para o arranjo do tipo idosa sozinha (de $1,1 \%$ para $18,1 \%$ ).

TABELA 1

Distribuição dos domicílios com idosos, por quartil de renda per capita, segundo tipos de arranjo domiciliar

Brasil - 1985-2005

\begin{tabular}{|c|c|c|c|c|c|c|c|c|c|c|c|c|c|}
\hline \multirow{4}{*}{\multicolumn{2}{|c|}{$\begin{array}{l}\text { Tipos de arranjos } \\
\text { domiciliares dos } \\
\text { idosos }\end{array}$}} & \multirow{2}{*}{\multicolumn{4}{|c|}{1985}} & \multicolumn{8}{|c|}{ Em porcentagem } \\
\hline & & & & & & \multirow{2}{*}{\multicolumn{4}{|c|}{$\frac{1995}{\text { Quartil de renda }}$}} & \multirow{2}{*}{\multicolumn{4}{|c|}{$\frac{2005}{\text { artil de renda }}$}} \\
\hline & & \multicolumn{4}{|c|}{ Quartil de renda } & & & & & & & & \\
\hline & & 10 & $2^{\circ}$ & 3음 & $4^{0}$ & 10 & $2^{\circ}$ & 3웅 & 40 & 19 & $2^{0}$ & 30 & $4 \stackrel{0}{ }$ \\
\hline 1 & $\begin{array}{l}\text { Casal morando } \\
\text { sozinho }\end{array}$ & 10,4 & 14,1 & 13,0 & 17,4 & 9,5 & 15,4 & 15,6 & 19,2 & 8,5 & 17,6 & 19,3 & 21,1 \\
\hline 2 & $\begin{array}{l}\text { Casal + outro } \\
\text { parente }\end{array}$ & 5,1 & 3,0 & 2,8 & 2,3 & 4,7 & 3,6 & 2,6 & 2,6 & 5,1 & 3,3 & 2,8 & 2,3 \\
\hline 3 & Idoso sozinho & 0,7 & 5,8 & 5,6 & 4,5 & 1,4 & 5,6 & 6,4 & 5,0 & 1,3 & 9,2 & 5,2 & 6,7 \\
\hline 4 & $\begin{array}{l}\text { Idoso + outro } \\
\text { parente }\end{array}$ & 0,8 & 0,7 & 0,7 & 0,7 & 0,8 & 0,8 & 0,6 & 0,6 & 0,7 & 0,7 & 0,7 & 0,6 \\
\hline 5 & Idosa sozinha & 2,7 & 15,4 & 8,2 & 8,6 & 1,8 & 15,3 & 13,5 & 12,2 & 1,1 & 18,1 & 11,9 & 15,2 \\
\hline 6 & $\begin{array}{l}\text { Idosa + outro } \\
\text { parente }\end{array}$ & 6,3 & 3,4 & 3,5 & 2,7 & 6,2 & 4,3 & 3,4 & 3,2 & 6,9 & 4,1 & 4,0 & 3,5 \\
\hline 7 & Casal com filhos & 25,8 & 19,2 & 21,3 & 23,1 & 21,6 & 16,9 & 18,8 & 20,7 & 19,0 & 12,4 & 17,7 & 17,7 \\
\hline 8 & $\begin{array}{l}\text { Casal com filhos + } \\
\text { outro parente }\end{array}$ & 13,9 & 10,0 & 9,9 & 7,2 & 17,1 & 9,6 & 8,6 & 6,7 & 18,3 & 7,9 & 7,1 & 5,6 \\
\hline 9 & Idoso com filho & 1,6 & 1,8 & 2,3 & 2,2 & 2,1 & 1,7 & 2,0 & 2,1 & 2,1 & 1,6 & 2,0 & 2,2 \\
\hline 10 & $\begin{array}{l}\text { Idoso com filho + } \\
\text { outro parente }\end{array}$ & 2,0 & 1,1 & 1,2 & 1,0 & 2,2 & 1,1 & 1,0 & 1,0 & 2,6 & 1,0 & 1,0 & 0,7 \\
\hline 11 & Idosa com filhos & 6,2 & 7,0 & 8,5 & 7,4 & 6,8 & 7,5 & 8,4 & 9,2 & 7,0 & 7,7 & 10,8 & 9,1 \\
\hline 12 & $\begin{array}{l}\text { Idosa com filho + } \\
\text { outro parente }\end{array}$ & 8,3 & 5,2 & 4,8 & 3,5 & 10,7 & 6,9 & 5,4 & 4,0 & 13,9 & 7,8 & 6,2 & 4,0 \\
\hline 13 & $\begin{array}{l}\text { Homem solteiro } \\
\text { com parente idoso }\end{array}$ & 1,2 & 1,9 & 2,7 & 2,6 & 1,3 & 1,4 & 2,1 & 2,2 & 1,2 & 1,2 & 1,7 & 1,9 \\
\hline 14 & $\begin{array}{l}\text { Mulher solteira } \\
\text { com parente idoso }\end{array}$ & 3,0 & 2,7 & 3,4 & 4,0 & 3,6 & 2,6 & 3,0 & 3,6 & 3,8 & 2,7 & 3,8 & 3,7 \\
\hline 15 & $\begin{array}{l}\text { Casal com parente } \\
\text { idoso }\end{array}$ & 11,9 & 8,8 & 12,0 & 12,9 & 10,3 & 7,3 & 8,5 & 7,9 & 8,6 & 4,6 & 5,9 & 5,7 \\
\hline \multicolumn{2}{|c|}{ Total } & \multicolumn{4}{|c|}{$100,0100,0100,0100,0$} & \multicolumn{4}{|c|}{$100,0100,0100,0100,0$} & \multicolumn{4}{|c|}{$100,0100,0100,0100,0$} \\
\hline
\end{tabular}

Fonte: IBGE. Pesquisa Nacional por Amostra de Domicílios - PNAD 1985, 1995 e 2005.

Essa enorme variação, do $1^{0}$ para o $2^{0}$ quartil, é explicada pelo recebimento de uma renda equivalente a um SM, pois é exatamente no $2^{\circ}$ quartil que se encontra esse valor, em qualquer um dos anos analisados. Assim, no 2ํquartil concentram-se os domicílio de idosos cuja composição da renda é fortemente determinada pelo recebimento de um salário mínimo, podendo ser na forma de uma aposentadoria, pensão, renda mensal vitalícia ou o BPC. Essa renda, supostamente, deve conferir maior autonomia ao idoso para decidir sobre seu arranjo domiciliar, ficando evidente a importância da renda no valor de um SM na determinação do arranjo domiciliar do tipo idoso sozinho (categoria 3), idosa sozinha (categoria 5 ) e casal morando sozinho (categoria 1). 
Outro resultado interessante é que, em todas as categorias nas quais há outro parente residindo no domicílio $(2,4,6,8,10$ e 12), quanto maior é a renda, menores são as proporções de domicílios nessa situação, sugerindo que corresidir com outras pessoas pode combater os efeitos da pobreza, sendo que a presença de outros parentes é um indicador dessa situação.

Apresentada essa seção descritiva, parte-se para a implementação do modelo de avaliação do impacto do BPC na determinação do arranjo domiciliar, considerando-se a importância da renda na determinação do arranjo domiciliar dos idosos. Assim, tanto a possibilidade de o idoso morar sozinho quanto a de agregação de familiares em torno da nova renda recebida podem ser afetadas pelo recebimento do BPC, como será visto a seguir.

\section{Resultados do impacto do BPC sobre o arranjo domiciliar dos idosos}

\section{Análise descritiva dos beneficiários do BPC vis-à-vis os não beneficiários}

A Tabela 2 apresenta resultados da análise descritiva dos grupos controle e de tratamento das variáveis relacionadas a características individuais e à localização geográfica dos residentes selecionados para a análise, onde se verifica que os indivíduos dos grupos controle e de tratamento são bem parecidos.

TABELA 2

Variáveis descritivas selecionadas dos idosos, por grupos de idade e situação de recebimento do BCP Brasil - 2002-2004

\begin{tabular}{|c|c|c|c|c|}
\hline \multirow[b]{2}{*}{ Variáveis selecionadas } & \multicolumn{2}{|c|}{ 63-64 anos (1) } & \multicolumn{2}{|c|}{$65-66$ anos (2) } \\
\hline & $\begin{array}{l}2002 \text { (não } \\
\text { receberam } \\
\text { BPC) }\end{array}$ & $\begin{array}{l}2004 \text { (não } \\
\text { receberam } \\
\text { BPC) }\end{array}$ & $\begin{array}{l}2002 \text { (não } \\
\text { receberam } \\
\text { BPC) }\end{array}$ & $\begin{array}{c}2004 \\
\text { (receberam } \\
\text { BPC) }\end{array}$ \\
\hline \multirow{2}{*}{ Sexo (porcentagem de homens) } & 47,37 & 42,86 & 52,02 & 47,15 \\
\hline & $(0,5)$ & $(0,5)$ & $(0,5)$ & $(0,5)$ \\
\hline \multirow{2}{*}{ Raça (porcentagem de brancos) } & 38,85 & 37,23 & 40,71 & 40,09 \\
\hline & $(0,49)$ & $(0,48)$ & $(0,49)$ & $(0,49)$ \\
\hline \multirow{2}{*}{$\begin{array}{l}\text { Escolaridade (média de anos de } \\
\text { estudo) }\end{array}$} & 1,82 & 1,88 & 1,76 & 1,87 \\
\hline & $(2,61)$ & $(2,9)$ & $(2,53)$ & $(2,64)$ \\
\hline \multirow{2}{*}{$\begin{array}{l}\text { Situação censitária (porcentagem de } \\
\text { residentes no meio urbano) }\end{array}$} & 75,39 & 76,29 & 77,71 & 76,73 \\
\hline & $(0,43)$ & $(0,43)$ & $(0,42)$ & $(0,42)$ \\
\hline \multirow{2}{*}{$\begin{array}{l}\text { Grande Região (porcentagem de } \\
\text { residentes nas Regiões Sul e Sudeste) }\end{array}$} & 32,66 & 32,67 & 35,38 & 36,04 \\
\hline & $(0,47)$ & $(0,47)$ & $(0,48)$ & $(0,48)$ \\
\hline \multirow{2}{*}{$\begin{array}{l}\text { Região Metropolitana (porcentagem } \\
\text { de residentes em área metropolitana) }\end{array}$} & 28,95 & 28,57 & 25,53 & 29,73 \\
\hline & $(0,45)$ & $(0,45)$ & $(0,44)$ & $(0,46)$ \\
\hline
\end{tabular}

Fonte: IBGE. Pesquisa Nacional por Amostra de Domicílios - PNAD 2002 e 2004

(1) Grupo controle que corresponde aos idosos que não receberam BPC em 2002 e 2004.

(2) Grupo de tratamento que corresponde aos idosos que não receberam BPC em 2002 e, por conta da mudança da legislação, receberam em 2004.

Nota: Desvios-padrões em parênteses. 
A Tabela 3 traz os resultados da análise descritiva da composição do arranjo domiciliar dos beneficiários e não beneficiários do BPC. São utilizados os termos "efeito idade" para designar a diferença entre os grupos 63-64 anos e 65-66 anos, quando se supõe que a diferença encontrada entre estes dois grupos deve-se apenas à idade, enquanto o termo "efeito BPC" é empregado para designar a diferença entre esses mesmos dois grupos ocorrida devido ao choque de renda desse benefício.

TABELA 3

Distribuição dos idosos, por grupos de idade e situação de recebimento do BPC, segundo tipos de arranjos domiciliares

Brasil - 2002-2004

\begin{tabular}{|c|c|c|c|c|c|c|c|c|c|c|c|}
\hline \multirow{3}{*}{\multicolumn{2}{|c|}{$\begin{array}{l}\text { Tipos de } \\
\text { arranjos } \\
\text { domiciliares }\end{array}$}} & \multicolumn{5}{|c|}{2002} & \multicolumn{5}{|c|}{2004} \\
\hline & & \multicolumn{2}{|c|}{$\begin{array}{c}\text { 63-64 anos (1) } \\
\text { (não receberam } \\
\text { BPC) }\end{array}$} & \multicolumn{2}{|c|}{$\begin{array}{c}\text { 65-66 anos (2) } \\
\text { (não receberam } \\
\text { o BPC) } \\
\end{array}$} & \multirow{2}{*}{ 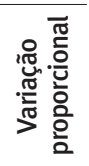 } & \multicolumn{2}{|c|}{$\begin{array}{c}\text { 63-64 anos (1) } \\
\text { (não receberam } \\
\text { BPC) }\end{array}$} & \multicolumn{2}{|c|}{$\begin{array}{c}\text { 65-66 anos (2) } \\
\text { (receberam o } \\
\text { BPC) }\end{array}$} & \multirow{2}{*}{ 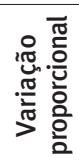 } \\
\hline & & N. abs. & $\%$ & N. abs. & $\%$ & & N. abs. & $\%$ & N. abs. & $\%$ & \\
\hline 1 & $\begin{array}{l}\text { Idosos(as) } \\
\text { sozinhos(as) }\end{array}$ & 38.838 & 13,8 & 41.093 & 14,9 & 8,0 & 36.380 & 12,1 & 57.382 & 19,4 & 61,0 \\
\hline 2 & $\begin{array}{l}\text { Idosos(as) } \\
\text { casados(as) } \\
\text { sem filhos } \\
\text { e outros } \\
\text { parentes }\end{array}$ & 40.089 & 14,2 & 44.076 & 16,0 & 12,0 & 48.222 & 16,0 & 44.764 & 15,1 & $-5,0$ \\
\hline 3 & $\begin{array}{l}\text { Idosos(as) } \\
\text { não } \\
\text { casados(as) } \\
\text { com filhos } \\
\text { e outros } \\
\text { parentes }\end{array}$ & 74.990 & 26,6 & 75.676 & 27,4 & 3,0 & 91.382 & 30,3 & 89.030 & 30,1 & $-1,0$ \\
\hline 4 & $\begin{array}{l}\text { Idosos(as) } \\
\text { casados(as) } \\
\text { com filhos } \\
\text { e outros } \\
\text { parentes }\end{array}$ & 127.740 & 45,4 & 114.936 & 41,7 & $-8,0$ & 125.135 & 41,6 & 104.387 & 35,3 & $-15,0$ \\
\hline \multicolumn{2}{|c|}{ Total } & \multicolumn{2}{|c|}{$281.657100,0$} & \multicolumn{2}{|c|}{$275.781100,0$} & - & 301.119 & 100,0 & 295.563 & 100,0 & - \\
\hline
\end{tabular}

Fonte: IBGE. Pesquisa Nacional por Amostra de Domicílios - PNAD 2002 e 2004.

(1) Grupo controle que corresponde aos idosos que não receberam BPC em 2002 e 2004.

(2) Grupo de tratamento que corresponde aos idosos que não receberam BPC em 2002 e, por conta da mudança da legislação, receberam em 2004.

Nota: A categoria "idosos(as) não casados(as) com filhos e outros parentes" corresponde à soma das categorias "idosos(as) não casados(as) com filhos" e "idosos(as) não casados(as) com filhos e outros parentes". O mesmo vale para a categoria "idosos(as) casados(as) com filhos e outros parentes".

A partir dos resultados da Tabela 3, destacam-se dois pontos em especial. Em primeiro lugar, observa-se que, em 2002, a variação entre os idosos de 63-64 anos e os de 65-66 anos que moram sozinhos (categoria 1) foi de $8 \%$, uma vez que passou de $13,8 \%$ para $14,9 \%$. A suposição feita é de que isso se deve unicamente ao efeito idade. Uma maior proporção de idosos morando sozinhos na idade de 65-66 anos do que na de 63-64 anos revela, supostamente, uma maior chance de os filhos terem saído da casa dos pais e de o cônjuge ter falecido. Já a diferença entre esses grupos em 2004 foi expressivamente maior (61\%), uma vez que passou de $12,1 \%$ para $19,5 \%$, o que leva a se supor que tal resultado 
deva-se ao efeito BPC somado ao efeito idade, assumindo-se que não tenha ocorrido, no período, outros choques de qualquer natureza.

Em segundo lugar, observa-se, na categoria idoso casado com filhos e outros parentes, uma variação negativa de 8\% no que tange ao "efeito idade" entre os grupos de 63-64 anos e 65-66 anos, em 2002. Esse resultado é coerente com a hipótese anteriormente referida de que, conforme aumenta a idade dos idosos, maior é a chance de seus filhos terem saído de casa e de já não haver mais um cônjuge compartilhando o domicílio. Ao analisar esses idosos em 2004, verifica-se que a variação encontrada aumenta para 15\%, o que corrobora o resultado anterior de que o recebimento do BPC concorre para a redução da proporção de idosos que vivem em domicílios mais numerosos (nesse caso, com cônjuges, além de filhos e outros parentes).

Essa análise inicial chama atenção, principalmente, para o possível impacto do BPC sobre os domicílios de idosos morando sozinhos. Isso é coerente com o resultado da composição domiciliar por quartil de renda, que evidenciou a importância de um rendimento no valor de um SM sobre o arranjo domiciliar independente. O que se supõe é que os idosos recebedores do $\mathrm{BPC}$ somam-se àqueles que, devido ao recebimento de um rendimento mínimo, puderam optar por um arranjo que prezasse pela privacidade.

\section{Resultado da análise do método diferença em diferença}

A análise segue agora mais aprofundada com os testes estatísticos do modelo e de significância. Será analisado o impacto do BPC sobre as seguintes variáveis respostas indicadoras de composição domiciliar: idosos(as) chefes morando com familiares (sejam eles cônjuges, filhos ou outros parentes de qualquer idade); idosos(as) morando na presença de filhos menores de 21 anos em domicílios cujo chefe ou cônjuge são idosos; idosos(as) morando na presença de outros parentes de qualquer idade em domicílios cujo chefe ou cônjuge são idosos; e tamanho do domicílio menor ou maior que três habitantes em domicílios cujo chefe ou cônjuge são idosos.

Em primeiro lugar, foi analisado o impacto do BPC sobre a chance de o idoso morar com familiares (sejam eles cônjuges, filhos ou outros parentes de qualquer idade). A averiguação do impacto é dada, na Tabela 4, pelo coeficiente da variável interação $\left(\beta_{3}\right)$, que, conforme discutido anteriormente, representa o impacto do recebimento do BPC sobre as variáveis respostas analisadas.

Nos três modelos estimados, observa-se que o coeficiente de interação (que fornece o resultado do impacto) é estatisticamente significativo (Tabela 4). 0 resultado indica que, excluindo-se essas diferenças, o impacto líquido do BPC sobre a chance de os idosos chefes morarem com familiares (sejam eles cônjuges, filhos ou outros parentes de qualquer idade) foi negativo e estatisticamente significativo. Assim, o recebimento do benefício, propiciado pela mudança na legislação, provocou uma redução na chance de os idosos chefes morarem com familiares (sejam eles cônjuges, filhos ou outros parentes de qualquer idade). Ou, em outras palavras, aumentou a chance de os idosos morarem sozinhos. 
Além do resultado do impacto do BPC, a análise das variáveis independentes do modelo permite fazer inferências sobre os arranjos domiciliares. Os modelos indicam que ser da raça/ cor branca ou amarela, pertencer à área urbana, residir na Região Sul ou Sudeste e ter boas condições de escoamento do lixo diminuem a chance de o idoso chefe morar com familiares, enquanto maior escolaridade aumenta essa chance.

\section{TABELA 4}

Estimativas dos modelos de regressão logística, cuja variável dependente é idosos(as) chefes que moram com familiares (sejam eles cônjuges, filhos ou outros parentes de qualquer idade) e cujo coeficiente da variável interação $\left(\beta_{3}\right)$ fornece o resultado da análise do impacto do recebimento do BPC sobre a chance de os idosos corresidirem com familiares

Brasil - 2002-2004

\begin{tabular}{|c|c|c|c|c|c|c|}
\hline \multirow{2}{*}{$\begin{array}{l}\text { Variável independente: } \\
\text { Idosos(as) chefes que moram } \\
\text { com familiares } \\
\text { Interação }(\beta 3)\end{array}$} & \multicolumn{2}{|c|}{ Modelo 1} & \multicolumn{2}{|c|}{ Modelo 2} & \multicolumn{2}{|c|}{ Modelo 3} \\
\hline & $-0,45$ & $(0,22)^{\star \star}$ & $-0,46$ & $(0,22)^{\star \star}$ & $-0,49$ & $(0,23)^{\star \star}$ \\
\hline Ano & 0,13 & $(0,17)$ & 0,13 & $(0,17)$ & 0,13 & $(0,17)$ \\
\hline Tratamento & $-0,08$ & $(0,16)$ & $-0,07$ & $(0,16)$ & $-0,04$ & $(0,16)$ \\
\hline Sexo & & & 0,06 & $(0,11)$ & 0,07 & $(0,11)$ \\
\hline Raça & & & $-0,25$ & $(0,11)^{\star \star}$ & $-0,2$ & $(0,12)$ \\
\hline Escolaridade & & & 0,06 & $(0,02)^{\star \star}$ & 0,06 & $(0,02)^{\star \star}$ \\
\hline Telhado & & & & & $-0,08$ & $(0,34)$ \\
\hline Paredes & & & & & 0,47 & $(0,23)^{\star \star}$ \\
\hline Banheiro & & & & & 0,23 & $(0,21)$ \\
\hline Água encanada & & & & & 0,48 & $(0,17)^{\star}$ \\
\hline Lixo & & & & & $-0,43$ & $(0,25)^{\star \star \star}$ \\
\hline Iluminação & & & & & 0,36 & $(0,27)$ \\
\hline Situação censitária & & & & & $-0,72$ & $(0,19)^{\star}$ \\
\hline Região metropolitana & & & & & 0,02 & $(0,13)$ \\
\hline Grande região & & & & & $-0,45$ & $(0,13)^{\star}$ \\
\hline
\end{tabular}

Fonte: IBGE. Pesquisa Nacional por Amostra de Domicílios - PNAD 2002 e 2004. Foi utilizada regressão da equação (2).

Nota: ${ }^{* *}$ significativo a $10 \%$, ${ }^{* *}$ significativo a $5 \%$ e * significativo a $1 \%$.

Os desvios-padrões estão em parênteses.

Com relação à presença de filhos maiores de 21 anos no domicílio (dados não apresentados), os resultados apontam um coeficiente de interação estatisticamente significativo. O sinal negativo do coeficiente indica que a chance de os idosos terem filhos no domicílio é menor para o grupo que recebeu o BPC.

Na investigação do impacto do BPC sobre a presença de outros parentes corresidindo com os idosos (dados não apresentados), o termo de interação também se mostrou negativo e estatisticamente significativo. Esse resultado indica que a chance de os idosos terem outros parentes no domicílio diminui se o individuo é do grupo de tratamento, ou seja, o recebimento da transferência reduz a chance de os idosos recebedores do BPC terem outros parentes no domicílio.

Por último, os resultados do coeficiente de interação da regressão da variável tamanho do domicílio maior que três moradores (dados não apresentados) apontam um coeficiente não estatisticamente significativo. 
Como se pode verificar, os resultados são todos consistentes e indicam que o recebimento da renda referente ao BPC reduz a chance de o idoso morar com familiares, ou, em outras palavras, aumenta a chance de o idoso morar sozinho.

\section{Considerações finais}

A literatura da demografia da família e dos domicílios está repleta de estudos empíricos que atestam o crescimento da proporção de idosos vivendo sozinhos ou apenas com seus cônjuges na Europa e nos Estados Unidos, sendo este também um fenômeno crescente nos países de desenvolvimento tardio (PALLONI, 2000; GARCIA; ROJAS, 2001; KLINENBERG, 2012). A questão de como a renda interfere nessa tendência em situações de elevada pobreza não é muito clara, uma vez que, de um lado, a autonomia financeira do idoso é condição essencial para viabilizar a busca de privacidade e, de outro, a maior segurança financeira do idoso pode ser um atrativo para parentes próximos. No caso específico do Brasil, a pergunta que se coloca é se as políticas sociais recentes, que tiveram como efeito a mitigação da pobreza dos idosos, produziram também o reforço da tendência de os idosos viverem sozinhos.

Nesse sentido, o trabalho investigou as duas perspectivas levantadas na literatura sobre o tema: o recebimento de uma renda tanto permitiria ao idoso optar pela privacidade no seu arranjo, como tornaria a corresidência atrativa para os familiares. Os resultados deste trabalho corroboram aqueles encontrados por Costa (1999), Mcgarry e Schoeni (1998) e Carvalho (2000), sustentando a hipótese de um efeito positivo de um choque exógeno de renda sobre a probabilidade de um idoso viver sozinho.

Já na análise descritiva da composição dos arranjos domiciliares segundo grupos de renda, fica claro o aumento da prevalência de idosos morando sozinhos entre aqueles que recebem rendimentos no valor de um salário mínimo.

A análise econométrica de impacto do BPC, utilizando o método estatístico da "diferença em diferença” e os dados da PNAD de 2002 e 2004, mostrou, também, que o BPC aumenta a chance de o idoso morar sozinho. Os resultados são bastante plausíveis, uma vez que os idosos analisados foram aqueles “mais jovens”, ou seja, pessoas cujo estado de saúde, de forma geral, permite a opção pela privacidade e independência no arranjo domiciliar. Além disso, o BPC é uma renda não contributiva que funciona como um choque exógeno de renda, destinado ao idoso que provavelmente se imaginava dependendo financeiramente de algum parente por toda a sua velhice. Dessa forma, supõe-se que o poder de decisão do idoso se modifica completamente com o recebimento da renda, viabilizando sua independência.

De maneira geral, a contribuição desse trabalho é demonstrar que, no contexto da implementação do BPC no Brasil, o efeito do choque exógeno de renda sobre os idosos pobres foi o aumento da formação de arranjos unipessoais. O entendimento desse fenômeno deve auxiliar a compreensão das tendências dos arranjos domiciliares no futuro e a elaboração e execução de políticas sociais, em que pesem as particularidades do caso analisado. Uma questão que se coloca é que, se para os idosos mais jovens o recebimento 
da renda significa maior chance de viver só, à medida que a idade avança e a dependência de cuidados torna-se maior, esse resultado pode ser invertido. De toda forma, se, de fato, uma renda nos moldes do BPC aumenta a chance de o idoso morar sozinho, é preciso pensar políticas complementares de assistência e cuidados pessoais aos idosos, uma vez que eles vão envelhecer e provavelmente acumular incapacidades.

Finalmente, para a melhor compreensão dos resultados encontrados, outros aspectos relacionados ao idoso devem ser incluídos em estudos futuros. A saúde do idoso e a sua idade têm grande importância na determinação da composição domiciliar do idoso. Idosos saudáveis podem exercer suas preferências, enquanto os debilitados, certamente, necessitarão de cuidados. O mesmo vale para a idade. Idosos jovens provavelmente apresentarão melhores condições de saúde, enquanto os mais velhos tendem a acumular mais incapacidades. Outro fator importante a ser considerado é a questão do gênero. Uma análise diferenciada para os arranjos domiciliares de homens e mulheres idosos é fundamental, pois os homens têm menor sobrevida e maior probabilidade de um recasamento do que as mulheres, no caso de viuvez ou divórcio. Somada a esses fatores, a maior autonomia financeira recentemente conquistada pela mulher faz com que o crescimento acentuado dos domicílios unipessoais de idosos que se vê hoje ao redor do mundo seja um fenômeno fundamentalmente feminino, cujas consequências ainda devemos conhecer melhor.

\section{Referências}

BRASIL. Lei n. 8.213, de 24 de julho de 1991. Dispõe sobre os Planos de Benefícios da Previdência Social e dá outras providências. Diário Oficial [da República Federativa do Brasil], Poder Executivo, Brasília, DF, 25 de julho de 1991. Coluna 2, p. 14.809.

Lei n. 8.742, de 7 de dezembro de 1993. Dispõe sobre a organização da Assistência Social e dá outras providências. Diário Oficial [da República Federativa do Brasil], Poder Executivo, Brasília, DF, 8 de dezembro de 1993. Coluna 1, p. 18.769.

Lei n. 10.741, de 1ํ de outubro de 2003. Dispõe sobre o Estatuto do Idoso e dá outras providências. Diário Oficial [da República Federativa do Brasil], Poder Executivo, Brasília, DF, 3 de outubro de 2003. Coluna 1, p. 1.

Ministério da Previdência Social. Base de dados históricos do Anuário Estatístico da

Previdência Social - AEPS Infologo. Brasília, 2006. Disponível em: 〈http://www3.dataprev.gov.br/ infologo/>. Acesso em: 20 jan. 2008.

CAMARANO, A. A. Mulher idosa: suporte familiar ou agente de mudança? Estudos Avançados, v. 17, n. 49, p. 36-64, set./dez. 2003.

CAMARGOS, M.; MACHADO, C.; NASCIMENTO, R. A relação entre renda e morar sozinho para idosos paulistanos: 2000. Revista Brasileira de Estudos de População, v. 24, n. 1, p. 37-51, jun. 2007. Disponivel em: 〈http://www.scielo.br/pdf/rbepop/v24n1/03.pdf〉. Acesso em: 8 jan. 2007.

CARVALHO-FILHO, I. Income effects on living arrangements and relative well being on unmarried elderly women in Brazil. 2000. 123 f. Ph.D. (Thesis) - Massachusets Institute of Technology, 2000.

CIOFFI, S. População: globalização e exclusão. Famílias metropolitanas: arranjos familiares e condições de vida. In: ENCONTRO NACIONAL DE ESTUDOS POPULACIONAIS, 11, 1998, Caxambu. Anais... Belo Horizonte: Abep, 1998, p. 30. 
COSTA, D. A house of her own: old age assistance and the living arrangements of older nonmarried women. Journal of Public Economics, v. 72, p. 39-59, 1999.

EDMONDS, E.; MAMMEN, K.; MILLER, D. L. Rearranging the family? Income support and elderly living arrangements in a low-income country. Journal of Human Resources, v. 40, XL, p. 186-207, 2005.

ENGELHARD, G.; GRUBER, J.; PERRY, C. Social security and elderly living arrangements: evidence from the social security notch. Journal of Human Resources, v. 40, 2005.

GARCIA, B.; ROJAS, O. Recent transformations in Latin American families: a sociodemographic perspective. In: XXIV GENERAL POPULATION CONFERENCE OF IUSSP, 2001.

GHOSH, S. Living arrangements among elderly women in India. In: SEMINAR ON AGEING IN DEVELOPING COUNTRIES: BUILDING BRIDGES FOR INTEGRATED RESEARCH AGENDAS. Anais... Santiago, Chile, 2007. Disponível para assinantes em: 〈http://www.iussp.org/members/restricted/publications/Santiago07/ programme07.php>. Acesso em: 26 jun. 2008.

GRUNDY, E. Living arrangements and the health of older in developed countries. In: UNITED NATIONS. Department of Economic and Social Affairs, Populacion Division. United Nations technical meeting on population ageing and living arrangements of older persons: critical issues and policy responses. New York: United Nations, 2000, p. 10.1-10.29.

IBGE. Pesquisa Nacional por Amostra de Domicílio (PNAD): microdados. Rio de Janeiro: IBGE, 1985, 1995, 2002, 2004 e 2005.

Diretoria de Pesquisas. Coordenação de População e Indicadores Sociais. Projeção da populaçao do Brasil por sexo e idade para o período 1980-2050 - Revisão 2008. Rio de Janeiro, 2008. Disponível em: 〈http://www.ibge.gov.br/home/estatistica/populacao/projecao_da_ populacao/2008/projecao.pdf〉. Acesso em: 17 mar. 2012.

KLINENBERG, E. Going Solo: the extraordinary rise and surprising appeal of living alone. Penguin Group, US, 2012.

MANNING, W. D.; BROWN, S. L. The demography of unions among older americans, 1980-present: family change approach. In: SETTERSTEN, R.; ANGEL, J. (Eds.). Handbook of sociology of aging. New York, Springer, 2011.

MARTELETO, L. Demographic change and the living arrangements of the elderly: the case of Brazil. In: POPULATION ASSOCIATION OF AMERICA (PAA) ANNUAL MEETING. Proceedings... Nova York, 2007. Disponivel em: 〈http://paa2007.princeton.edu/download.aspx?submissionld=71698〉. Acesso em: 24 jun. 2008.

MCGARY, K.; SCHOENI, R. Social security, economic growth, and the rise in independence of elderly widows in the 20th century. Cambridge, Mass: National Bureau of Economic Research, 1998 (Working paper, 6511). Disponivel em: 〈http://www.nber.org/papers/w6511〉. Acesso em: 24 abr. 2007.

MIRANDA, V. Public transfer and interhousehold transfers to the elderly in Brazil. In: POPULATION ASSOCIATION OF AMERICA (PAA) ANNUAL MEETING. Proceedings... New Orleans, 2008. Disponível em: 〈http://paa2008. princeton.edu/sessionViewer.aspx?sessionld=951〉. Acesso em: 01 out. 2008.

PALLONI, A. Living arrangements of older person. Center for Demography and Ecology, University of Wisconsin-Madison, Feb. 2000 (CDE Working paper, 2000-02).

RAHMAN, M. O. Living arrangements and the health of older persons in less developed countries: evidence from rural. Bangladesh. In: UNITED NATIONS. Department of Economic and Social Affairs. Populacion Division. United Nations technical meeting on population ageing and living arrangements of older persons: critical issues and policy responses. New York: United Nations, 2000, p. 11.1-11.20.

SAAD, P. M. Impact of pension reform on the living arrangements of older persons in Latin America. In: UNITED NATIONS. Department of Economic and Social Affairs. Populacion Division. United Nations 
technical meeting on population ageing and living arrangements of older persons: critical issues and policy responses. New York: United Nations, 2000, p. 6.1-6.12.

SOARES, F.; SOARES, S.; MEDEIROS, M.; OSÓRIO, R. Programas de transferência de renda no Brasil: impactos sobre as desigualdade. In: ENCONTRO NACIONAL DE ECONOMIA, 34, 2006, Salvador, BA. Anais... Belo Horizonte: Anpec, 2007. Disponivel em: 〈http://www.anpec.org.br/encontro2006/artigos/ A06A156.pdf〉. Acesso em: 15 jul. 2007.

\section{Autoras}

Maira Andrade Paulo é doutora em Demografia e professora de Economia na Universidade Salgado de Oliveira.

Simone Wajnman é doutora em Demografia e professora do Departamento de Demografia da Universidade Federal de Minas Gerais - UFMG.

Ana Maria Camilo Hermeto de Oliveira é doutora em Demografia e professora do Departamento de Economia da Universidade Fedral de Minas Gerais - UFMG.

\section{Abstract \\ The relationship between income and living arrangements of elderly persons in Brazil: the impact of the Continuous Cash Benefit Transfer Program (BPC)}

This paper analyzes the impact of income for the elderly from the Continuous Cash Benefit Transfer Program (BPC) on the living arrangements of elderly poor persons. This benefit consists of income received monthly, equivalent to one minimum wage, by elderly persons over age 65 whose monthly per capita family income is below one-fourth of the minimum wage. This discussion is relevant in the current Brazilian context, which has been marked by rapid population aging, broad changes in living arrangements and an expansion of social security benefits for the elderly. Two hypothesis discussed in the literature were investigated, using the difference-in-difference statistical method and based on data from the 2002 and 2004 PNADs. It was seen that this unconditional income transfer would either (i) increase the probability of the elderly persons involved to live independently, or (ii) increase the probability of co-residence with family due to the attraction exerted by this additional income. The results obtained show that the receiving of the BPC income increases the probability that beneficiaries will tend to seek independents living arrangements.

Keywords: Income transfer. Living arrangements of the elderly. Continuous Cash Benefit.

\section{Resumen}

La relación entre ingresos y composición domiciliaria de los ancianos en Brasil: un estudio sobre el impacto de la recepción del Beneficio de Prestación Continuada

Este trabajo analiza el impacto de la recepción del Beneficio de Prestación Continuada (BPC) sobre la composición de los arreglos domiciliarios de los ancianos pobres en Brasil. El BPC corresponde al beneficio mensual de un salario mínimo (SM) destinado a personas con más de 65 años, cuyos ingresos familiares per capita sean inferiores a 1/4 del SM. Esta discusión es bastante oportuna en el actual contexto brasileño, marcado por un acelerado envejecimiento poblacional, por profundos cambios en los arreglos domiciliarios y por la amplia cobertura de seguridad social del anciano. Utilizando el 
método estadístico de la "diferencia en diferencia" y los datos del PNAD de 2002 y 2004, se investigan dos hipótesis discutidas en la literatura sobre el tema: la recepción de una transferencia incondicional de renta elevaría la probabilidad de que los ancianos vivieran solos, en función de la autonomía otorgada por los ingresos, o aumentaría la probabilidad de la co-residencia con familiares debido a la atracción ejercida por la renta. El resultado sugiere la hipótesis de que la recepción del BPC, en Brasil, ha aumentado la probabilidad de formación de domicilios unipersonales.

Palabras clave: Arreglos domiciliario de los ancianos. Transferencia de ingresos. Beneficio de Prestación Continuada (BPC).

Recebido para publicação em 26/01/2012

Aceito para publicação em 27/03/2012 
